\title{
Patchwork quilt or woven cloth? The student experience of coping with assessment across disciplines
}

\section{Author: Berry O’Donovan}

\author{
Abstract \\ This paper explores assessment experiences of undergraduates studying across disciplines. Within a \\ participatory research design students as researchers were involved in data collection and \\ interpretation. The student lens is brought to bear on the experiences of academically successful final-year \\ students and their strategies for negotiating assessment across disciplinary departments. Findings highlight \\ perceptions of invisibility, academic homelessness and disadvantage despite participants' high academic \\ achievement. These successful students viewed the divergent disciplinary approaches to assessment they \\ encountered as legitimate, but felt challenged and disadvantaged by their diversity. Findings are discussed \\ within a framing of assessment practice as socially situated and embedded in the educational values and \\ epistemologies of learning communities, thus problematizing development of student assessment literacy \\ across disciplinary communities. The paper concludes that it is neither feasible nor educationally valuable \\ to iron out divergence but more intentional ways of revealing and sharing different epistemological \\ positions in assessment practice could mitigate challenges.
}

Keywords: Assessment, Assessment literacy, Discipline, Multidisciplinary, Epistemology

\section{Introduction}

The experience of studying across multiple disciplines can accelerate student learning and capability to deal with the complex, multidisciplinary issues of today (Feng 2012). In the Americas the Society for University College and Planning (SCUP) points to an 'Increasing recognition that the future of learning is multidisciplinary or cross disciplinary' (SCUP Academy 
Council 2014, 8). However, researchers suggest that the experience can also be challenging, as students have to learn to work within two (or more) academic cultures and ways of knowing (Timmis and Williams 2017; Canning 2005). Portmanteau degrees and modular structures have become ubiquitous across the international higher education sector, and many single honours degrees are essentially multidisciplinary, incorporating a range of disciplinary perspectives fragmented into discrete modules. However, the experience of students studying across disciplines remains under-researched and reported (Canning 2005; Weissmann 2013).

This paper explores the assessment experience of undergraduates in a UK university studying across two departments, on what are often interchangeably labelled interdisciplinary degrees, joint-honours or combined degrees. Such degrees generally involve the combination of two or three disciplines (e.g. economics and geography). Throughout this paper the term multidisciplinary is used as distinct from interdisciplinary, as the latter is generally understood as when two disciplines come together in an integrated form to create new knowledge that spans disciplinary boundaries (Lattuca 2001; Klein 1990). However, it is common under modular programmes that disciplinary areas and departments remain discrete, it is therefore argued that many such degrees are in reality multidisciplinary in nature, as in the context of this study.

Students studying across multiple disciplines must navigate across different ways of knowing mediated by discipline-based faculty. The challenge of successful navigation is especially acute in the context of assessment. Assessment distils the expectations and epistemic assumptions of disciplinary communities, requiring successful students to understand such assumptions and to operate effectively within them (O’Donovan 2017). The paper initially frames the research within the concepts of disciplinarity and assessment literacy. Subsequently, drawing on a 
participatory research approach using students as researchers involved in data collection and its interpretation, the assessment experience of academically successful students and their strategies for clarifying and managing assessment across disciplinary departments is thematically explored and discussed.

\section{Disciplines and disciplinary difference}

Klein (1990) suggests that the modern concept of disciplinarity became prevalent during the 19th century fuelled by the industrial revolution and an attendant commercial demand for specialist expertise. Today, most academics in higher education still operate within departmental structures, the organisational rationale for which is discipline-related (Mathieson 2012). However, the notion of 'discipline' is ambiguous. Much of the early literature is structuralist in nature, tending to abstract and de-contextualise components of disciplines, seeking to define and categorise their fundamental characteristics (see for instance Biglan 1973a, 1973b).

Poststructuralist accounts emphasise disciplines as socially situated and constructed as learners become progressively acculturated as they advance through education and are increasingly likely to interpret and make sense of information and their academic experience through a disciplinary lens (see for instance Becher and Trowler 2001).

Whilst the uniformity of disciplinary characteristics in all localised contexts is questioned (Trowler, 2014), most authorities agree that certain particularities of disciplines can be discerned. Under Biglan's seminal classification system (1973b), disciplines are categorised into two dimensions: hard-soft and pure-applied. Hard fields are viewed as those that value and strive for universal understandings that have developed over a long trajectory of research, often with a strong quantitative emphasis. Their knowledge structures tend to be hierarchical, linear and 
cumulatively layered. Neumann, Parry, and Becher $(2002,407)$ suggest that until an advanced stage of study, teaching within hard disciplines focuses 'on established facts and on demonstrable theories rather than uncertainties and relativities'. Conversely, soft fields are those that embrace multiple perspectives and ambiguity, encourage student engagement with divergent ideas and interpretations, and in which knowledge is rarely superseded by updated understandings. Knowledge is understood as 'qualitative, constructed and interpretive' (Jones 2007, 87). The pure-applied axes focus on the difference between pure fields that seek to discover and develop new theories and those more engaged with the application and use of knowledge.

Based on later research studies Braxton and Hargens $(1996,35)$ concluded that Biglan's work remained relevant and that disciplinary differences were 'profound and extensive'. Studies suggest that such differences acutely influence the epistemic beliefs of academic staff and their teaching and assessment methods (Becher and Trowler 2001; Neumann, Parry, and Becher 2002), and in turn the epistemic assumptions of students (Paulsen and Wells 1998). For example, studying within the context of a 'soft' discipline may encourage epistemic beliefs in the complexity and contestability of knowledge. Conversely the context of a 'hard' discipline may reinforce beliefs in the certainty and objectiveness of knowledge.

Here, a note of caution - later research suggests that such generalisations of disciplinary characteristics are overly simplistic and too starkly drawn. Trowler (2014) eruditely argues that such accounts are excessively essentialist, over exaggerating the homogeneity of core, disciplinary characteristics, and their influence on other phenomena (such as teaching and 
assessment). He suggests such strongly essentialist accounts mask a more complex and contradictory reality. Trowler (2014) and Mathieson (2012) view disciplinary cultures as socially situated, suggesting in combination with other influences - such as expectations of students, marketisation, globalisation, etc. - disciplines do influence academic culture and practice, but not homogeneously in all localised contexts. Using Wittgenstein's (1953) notion of 'family resemblances', Trowler (2014) suggests that disciplines may display characteristics that have a family resemblance, but not all family members will necessarily share all features.

\section{Assessment and assessment literacies}

There is convincing evidence that assessment is a key driver of student learning, and that the nature and form of assessment define the curriculum for students and powerfully influence their learning approaches and behaviours (see for instance Gibbs and Simpson 2005; Ramsden 1992). Assessment judgements and practices are situated, interpreted, and representative of the educational values of disciplinary learning communities within which assessment is enacted (Shay 2008). Indeed, Neumann, Parry, and Becher $(2002,408)$ assert that assessment practices illustrate the issues that academics 'consider particularly significant within their discipline, whether they be questions of approach and understanding or matters of factual content'.

Neumann, Parry, and Becher $(2002,408)$ describe differences between the typical assessment practices of disciplinary learning communities. They claim that in science-based hard-pure fields there is a tendency 'to prefer specific and closely focused examination questions to broader, essay-type assignments', in conjunction with an emphasis on objective testing in which the cumulative knowledge acquisition of students is frequently and comprehensively tested. By contrast in soft-pure subjects the goal of assessment is viewed as more likely to determine 
students' understanding of the multiple perspectives inherent in a subject domain along with their own perspectives on complex and debated issues. In soft-pure subjects coursework, including essays and oral presentations, is often undertaken throughout a semester, contrasting with the final summative examination of hard pure fields. In soft-applied fields project work and reflective writing is more likely to be emphasised including self and peer assessment practices to underpin the development of professional skills.

Sadler (1989, 2009) and Price et al. (2012) also highlight the social situatedness of assessment, particularly the importance of alignment between student and assessor understandings of the qualities of a good assessment response. They assert that an indispensable condition for students to do well, in whatever context they are assessed, is that the instructional design should enable students to understand the assessment standards and attributes of quality being sought. Price et al. $(2012,7)$ in developing the concept of assessment literacy stress that 'for students to reach their potential in terms of their assessed performance they need to become "assessment literate" in terms of developing an aligned understanding of assessment standards, criteria, techniques and the relationship of assessment to learning.' They suggest such assessment literacy is built up over time within a learning community. However, whilst they make many practical suggestions as to how assessment literacy can be developed through, for example, student-to-student and student-to-staff dialogue, marking practice, and peer and self-assessment there is little consideration for the challenges of studying across different disciplinary communities.

Many single honours degree programmes can be considered multidisciplinary 'fields of study' involving multiple assessment cultures and practices. Business, for example, draws from 
psychology, economics, sociology and statistics amongst others. It is not alone, the study of 'geography' incorporates hard and soft subjects from humanities and the physical and social sciences, the study of politics can involve history, economics, sociology, and so on. Such multiple disciplinary approaches can be more or less integrated across a field of study (Bernstein, 1996), although Hughes, Smith and Creese (2015) state the latter is more common, asserting that nowadays most undergraduate programme structures are fragmented into modules. Bernstein (1996) labels these collection-type curricula in which modules are highly differentiated and taught by diverse disciplinary specialists, or as Lattuca $(2001,11)$ describes them 'patchwork quilts' rather than 'woven cloths'. Accordingly, students who operate effectively across two or more disciplinary communities, successfully navigating their different assessment practices, will bring different assessment literacies to bear, not just in each field, but in each module. Successful accomplishment arguably requires ‘epistemic fluency’ which Morrison and Collins $(1996,109)$ define as the ability 'to identify and use different ways of knowing, to understand their different forms of expression and evaluation, and to take the perspective of others who are operating within a different epistemic framework'. However, whilst students undertaking multidisciplinary degrees have to negotiate between the different assessment literacies bound up in different disciplinary cultures and practices, this is arguably less the case for many academics who are likely these days to be more specialist (Macfarlane 2011) and embedded in departments, the underpinning organisational rationale for which is commonly the discipline (Lattuca 2001). Consequently, we can conjecture that such academics may have little understanding of the differences encountered by students in the multidisciplinary and modular programmes in which they teach. 


\section{Research Context and Methodology}

A participatory research approach was taken in this study so that data would both be drawn from student participants and predominantly analysed and interpreted through a student lens. To this

end, the research team comprised of two academics with considerable research experience within a pedagogic research centre but who also teach in a business school, and four student research assistants (SRAs). The latter were recruited into formal, part-time and paid roles under University human resource and research ethics regulations from multidisciplinary (combined honours) undergraduate degree courses. The four SRAs took part in data collection, analysis and interpretation. Bergold and Thomas (2012) state that participatory research methods are geared towards conducting research with people 'whose life-world and meaningful actions are under study'. Consequently, for many, participatory research embodies values of democratisation, involving collaboration with those who might otherwise be subjects of research or recipients of interventions arising from research. Welikala and Atkin $(2014,391)$ suggest that this is particularly important in pedagogic research as this is currently dominated by researchers interpreting the student experience, the inevitable result of which 'is the subtle enactment of researcher and teacher authority on the learner' experience'.

Initially, the project's intentions along with relevant assessment and disciplinary understandings drawn from the literature were shared and discussed with the SRAs. Based on these initial understandings the SRAs undertook further literature searches and readings, adding to the research team's knowledge base, and used to inform the design and preparation of participatory student and staff research workshops. Final-year, academically successful, combined-honours students were invited to participate in two extra curricula half-day 'workshops' incentivised by 
book vouchers. What is 'academically successful' is a matter of debate. This study adopted a largely instrumental approach, categorising this as the achievement of high grades. An aim being that findings might subsequently inform strategies to enhance the number of 'good degrees' (categorised as upper second and first class honours degree classifications in the UK) across multidisciplinary programmes.

Eleven students participated who were taking 'Business' with one other subject: Geography, International Relations; Law; Politics; Psychology; Publishing; Sports, Coaching and PE; Sociology; Spanish; Statistics. These students were supported to reflect on their assessment experience across their combined honours degree in collaboration with the SRAs who both participated within and facilitated the workshops. Workshop participants were encouraged to depict and discuss critical points in their assessment experience in a variety of ways, including consideration of the level of learning and assessment challenge across their degree subjects: recounting stories about, and visually mapping, critical points of difficulty, discomfort or success.

The data collection method intentionally incorporated both visual and verbal modes of investigation. Vaughan (2005) advocates such multiple modes for valuing and invoking multiple and distinctive understandings and ways of knowing. The workshops comprised recorded discussions with a loose framework of prompts to help centre discussions on participants' assessment experience, but discussions were wide-ranging embracing a broad network of factors that both directly and indirectly affected this experience. In twos or threes, participants were also encouraged to visually draw or map their assessment. Participants subsequently explained and 
discussed these in plenary with the SRAs who both facilitated and contributed to the discussions, noting down particular points of interest, divergence and similarity. The staff researchers attended to the audio recordings and photographed the visual artefacts.

The data from the visual maps and recordings were initially reviewed by the staff researchers who produced a broad analysis framework. Interestingly, Weller et al. (2013) state that even within participatory research frameworks the experience of students is often interpreted within researcher generated analytical frameworks. Five broad descriptive domains were presented in the analysis framework: community and identity; practicalities and infrastructure; academic issues; cognitive and affective experiences, and; other. These were discussed with the SRAs along with identifying possible examples of key issues drawn from the workshop data. Based on the analytical framework and close listening to the workshop recordings, alongside review of the visual maps, the SRAs each authored an analysis of the data, refining domains and drawing together expressions into broad categories and giving these conviction and voice through the use of multiple quotes. As the SRAs were combined honours students themselves they were asked to add a personal commentary on their experience of assessment across their chosen disciplines and suggestions for practical enhancements. In the last stage of the analysis the staff researchers reviewed the SRAs' analyses and their personal commentaries. The SRA's analyses were closely read and re-read and progressively interpreted into themes. Finally, themes were discussed and agreed with the SRAs as a group to support the dependability of analysis. Kvale describes this as 'agreement through rational discourse and reciprocal technique' $(1996,65)$. 


\section{Findings}

Themes demonstrate the interconnectedness of assessment with the wider learning and teaching experience. In discussions participants considered how a broader set of issues such as programme structures, belonging and identity directly or indirectly affected their assessment experience.

\section{Alienation and a sense of academic homelessness}

Combined honours students can feel lost between two departments and suffer from a relative lack of cohort identity and sense of belonging. Students expressed difficulty joining modules with single-honours students that they did not know. They felt challenged by constantly seeing new faces including new tutors every time they started a new module and this affected their confidence in terms of contributing effectively in class and undertaking assessment. Students felt overlooked in classes where seemingly tutors had little knowledge that there were combined honours students in the class.

...every module you do, it's always going to be new people, and it's harder [than studying within a single discipline] I think. (International Relations/Business)

I don't feel very connected to Sociology so I am more nervous about asking for help or guidance on assessment. (SRA Sociology/Business)

In terms of community, I can say I have truly struggled. Having taken the Introduction to Business and Management class and having the same classmates both semesters, I recognized faces and felt comfortable speaking up in lectures and seminars. In my 
Sociology modules, however, I didn't know a single face or name, and therefore felt incredibly uncomfortable participating in class discussions. Many people already knew each other and formed cliques, sticking together for classes, so I felt alienated and kept to myself. (Sociology/Business)

Students could not only feel academically homeless but invisible, expressing that their experience as studying across departments as not generally recognised or known to the institution.

Personally, I do not know whether I belong to the business faculty or the social sciences. It is hard to decide. When it comes to surveys, you can only choose one faculty. There is only ever space for one faculty in any evaluation form. Nobody understands you can belong to two. (Politics/Business)

If you don't make Publishing [The Publishing programme] your life, you feel a bit out of place in the department, they don't seem to know they have combined honours students. (Publishing/Business)

\section{A sense of academic disadvantage}

Students taking combined degrees felt at risk and at an academic disadvantage as they took fewer modules in each of their subjects and thereby felt they lacked prerequisite knowledge and assessment experience. This sense of academic disadvantage not only came from a felt lack of 
content knowledge but also the recognition that what was being sought from assessors differed from module to module and needed to be discovered afresh for each assessment.

...even though some modules do not require a prerequisite [prior module], some knowledge is expected anyway. (Geography/Business)

We don't have as much practice in writing business reports as the single honours students do. (Spanish/Business)

It is a module taken by finance students as well as ours, they already knew... once I had the language, and understood more the technical aspect I could run with it. (Business/Politics)

...because I'm majoring in Business, when I go across to Sports Coaching, everybody already knows the terminology, you have to play catch-up I find. (Sports/Business)

\section{Variation in assessment requirements and expectations}

Learning within two (or more) disciplines requires engagement with divergent ways of knowing in terms of the nature of knowledge, accepted methods, concepts, assessment standards and criteria, and so on. Students recognised this epistemic variation and expressed their experience of it in sophisticated ways. Nevertheless they resented the resultant challenge and lack of clarity on assessment expectations, standards and the attributes of a good assignment that this presented. In the workshop students reflected: 
In terms of the epistemological underpinnings of it, that's a module which is more positivist, you either got the answer right or you didn't... and if you didn't get the terminology you weren't going to have the right answer. (Business/Statistics)

I had a massive Foucauldian essay to do, and that's completely different from Business, I'd say really distinctly different. Once you have got your head around how to do this kind of essay it is difficult to remember the approach of a business report. (Business/Sports)

Workshop participants discussed assessment requirements and expectations and how these not only varied between the two disciplines of their degree, but also between modules within a discipline, and between tutors:

It is not just between fields [disciplinary departments] there are differences. There seems to be rampant confusion across all modules when it comes to assessment. Every module leader is looking for a different approach to a question, a different style of writing, a different viewpoint of the material. (Business/Spanish)

Creativity, a module I've just done, it's mind blowing because you have no idea what to do, there's no guidance in terms of marking grids. There isn't that, there's no guidance on how to get a good grade. (Business/Law) 
I had a 3rd year geography one [module] which I still don't have a clue what it's about ,and there was a 2000 word essay, and I think I got 68 on it and I honestly didn't have a clue what I was writing, I was expecting a 2:2. No one knew what was going on, it just wasn't logical. (Business/Geography)

\section{Zones of discomfort and academic challenge}

In twos or threes participants were asked to visualise their assessment experience using write-on tablecloths and marker pens. Groups independently visualised this in terms of a temporal journey in which diversity in expectations and requirements were mapped and highlighted both across years and fields of study but also modules. Modules may gain the same academic credit but students considered challenge differed markedly between individual modules. Critical moments of challenge were expressed as when expectations and requirements changed and new ways of knowing had to be understood and demonstrated. Most participants considered that variation between modules was at its peak in the second year of university, with the experience of academic challenge fluctuating between modules from manageable to overwhelming. For most the first year in which basic knowledge, academic conventions, techniques and skills and knowledge that could be learnt with application and memorisation was relatively straightforward.

The first year was straightforward, if you worked hard you got through your modules. (Business/Statistics)

However, one SRA working on the project was from Nigeria. In her report she reflected on another dimension of epistemology and expectations that related to being not only a combined 
honours student, but also an international student. Her comments suggest that they experience of challenge may follow a different trajectory for some international students:

Like many other students in the workshop, I felt that taking a joint honours course was a whole lot more challenging than single honours in terms of not only organising one's time, but having to constantly change your thinking style in each field. I share similar opinions in terms of lack of clarity in assessment criteria and the need to encourage more peer mentoring. However, my experience of university life is to an extent different from most student participants. I feel that as an international and mature student, first year was very challenging compared to second year. I had to try to understand the English way of learning, the language, the setting and the style. I was used to a different educational life where assessment is basically through exams and memorising was one of the ways forward. This is more reason why I sketched a map different from the others. (SRA, Business/International Relations).

However, this experience was not shared by two other international SRAs (American and Singaporean), both of whom had attended American high schools prior to higher education, a salutary reminder that the international student experience is not homogenous.

\section{Successful strategies to seek clarity on assessment expectations}

The academically successful students participating in the workshops actively found ways to gain clarity on expectations. Many of them proactively sought out teaching staff in their offices, participated in and valued informal peer study groups as well as formal processes in which they 
could read, review and discuss work in relation to their quality and standard (their own, each other's or exemplar assignments).

I learn through talking to people, and communicating with my peers, and through that process I get a much higher understanding of it, and then I'm able to reflect back on what I've learnt in my assignment. I ask tutors 'is that the right territory? (Business/Politics)

Customised grading rubrics, explicit instructions, and perhaps even example papers showing the difference between an A grade and D grade greatly benefits all parties involved. (Business/Geography)

Some students were also conscious that assessment expectations may differ between tutors, even those on the same module, explaining they had been caught out in the past:

We really struggled knowing what to include in our assignments and when we asked our seminar leader 'do we need to include this certain thing' and he said 'it's completely up to you'. However, when it came down to the assignment being marked some fundamental points were missing.

I email both the module leader and seminar leader [individually] my questions and check the responses against each other. (Business/Spanish) 


\section{Complex structures and processes require organisation and knowledge}

Workshop students discussed the need to ably navigate complex programme structures and processes to successfully organise their studies and assessment preparation and submission, a challenge compounded by staff with seemingly insufficient knowledge of the issues.

You have to know who to speak to, who can really help you make sure your programme is OK. (Business/Politics)

Sometimes because of schedule clashes you can't do a module you want to do, but often there is a way, you need to know who knows. (SRA Business/International Relations).

Workshop participants discussed how assessment deadlines can impact on combined honours students:

The departments don't talk to each other, nobody in Geography knows what Business does. At least Business makes an effort to understand the experience... But assessments can pile up across with no one knowing and it is very stressful. (Business/Geography)

Practicalities including inter-campus travel and different departmental processes could also be a source of confusion and stress.

It is not possible to travel between... [two different satellite campuses] ...within the one hour transfer time and it means I miss the beginning of lectures. (Business/Sports) 


\section{Discussion}

The SRAs and workshop participants were academically successful students who encountered challenges in coping with assessment and learning across multiple disciplines, but who ultimately thrived. Overall their assessment experiences were characterised by structural, cultural and epistemic diversity and dissonance. This may contrast with the experience of many academic staff. Becher and Trowler (2001) suggests that academics are likely to be more embedded in and committed to their own disciplinary tribe than either multidisciplinary institutional or course roles and contexts, even where disciplinary characteristics vary across localised contexts. This is not to suggest that disciplinary departments do not harbour dissenting subgroups with different ontological perspectives (Lattuca 2001). The many influences on academic ways of knowing and practicing should be acknowledged. Boyd and Smith (2016) highlight other important social and cultural influences including the orientation of an individual academic's work between teaching, research and administration. In addition, an increasingly managerial and commercial emphasis in higher education (O’Donovan, 2017) nowadays may (and may even seek to) diminish academic autonomy and the influence of the discipline on structures and processes. However, Boyd and Smith (2016) note the dangers of underplaying the significance of differences between subject disciplines and their effect on academic identities as well as organisational structures and processes.

Participants' dissonant experiences were often recounted at module level within a field of study perspectives and processes were not universal across a department let alone the institution. Arguably, this may partially be explained by the nature of the fields of study students were combining. The programme coherence of business, geography, politics, publishing, international 
relations, psychology, and sport sciences can all be questioned as they incorporate elements of different disciplines and departments. Such fields of study are inherently multidisciplinary, and combining two of these fields may merely exacerbate the diversity of ways of knowing and practising that students encounter. However, it is rare that in higher education academics teach across diverse subjects within a field of study. Indeed, Macfarlane (2011) suggests that academics have become increasingly specialised in terms of their discipline focus and their portfolio of activities.

Many authorities suggest as the discipline remains a key organising feature and influence on academic life this can pose challenges for academics to understand the student experience across a multidisciplinary programme (Weissmann 2013). Moore (2000) also suggests that strongly insulated academic tribal cultures and practices decrease the ability of academics to see beyond their own modules, particularly in collection-type curricula within which disciplinary-based modules remain insulated from each other. In such circumstances it is often left up to the student to make coherent sense of the whole. Findings from this study display how this can leave students feeling academically homeless, without the disciplinary identity that can provide academics with a sense of belonging. Indeed, the concept of the discipline as providing a sense of belonging and home for students in today's modular environment appears completely extraneous to the experience of these combined honours students who saw their degree at best as two 'halves' and at worst a jumble of dissonant modules. Some students described their experience not just as 'not belonging', but as one of isolation and alienation. One student expressed how 'invisible' combined honours students were within the institution. This is echoed by Weissmann (2013) who notes that single honour courses are considered the norm and 
assessment processes and their attendant quality assurance processes are generally orchestrated around them.

Pedagogy-informed assessment practices are deeply rooted in established disciplinary epistemologies (Moore 2000), and these are often implicit and unexpressed. O'Donovan, Price and Rust (2004) outline the tacit nature of assessment standards and marking criteria, and the challenges of sharing these in a meaningful way with students. Price et al. (2012) view assessment literacy as slowly learnt with understandings shared over time and involving student (and staff) participation within learning communities. The belief is that over time students will be socialised into a discipline, including the norms and tendencies involved in its assessment practice. In multidisciplinary contexts Lea and Street (2006) argue that successful students need to be able to nimbly switch practices between one context and another using different academic practices appropriate to each setting, and negotiate the social meanings and identities that each evokes. This resonates with Morrison and Collins (1996) notion of epistemic fluency conjectured as a key competency in successful multidisciplinary and interdisciplinary assessment and learning. Goodyear and Ellis $(2007,65)$ go further, arguing that students draw benefit from induction to more than one epistemic community, but found 'very few signs of teachers inducting students into ways of knowing and thinking characteristic of their discipline or profession'. Within this study it was left to the student to develop adaptive expertise extemporaneously rather than within a more intentional and planned, instructional course design.

The progressive development of students' assessment literacy is undeniably problematic in multidisciplinary contexts. O'Donovan (2017) notes students' reluctance to take forward 
assessment literacies gained in one module to another, commenting that this indeed may be risky in epistemologically diverse fields of study. Indeed Hughes, Smith and Creese (2015) state the assessment feedback to students is rarely authored by academics in ways to be applicable beyond module boundaries. In this study combined honours students recognised the patchwork nature of assessment practices and the fundamental assessment differences in play between their two fields of study. However, they felt at an academic disadvantage, not just because they came to modules with less content knowledge but also because they recognised and experienced the breadth and diversity of perspectives on assessment, particularly the attributes of quality of a good piece of work. Most participants viewed such differences as legitimate, but all desired enhanced clarity on assessor expectations to support the attainment of high marks. An instrumental objective that Dean and Gibbs (2015) note has intensified across higher education over the past decade.

Disciplinary ways of knowing validate particular ways of thinking and practicing, defining acceptable methods, concepts, and academic and assessment literacies. As such, coherent disciplines can limit the number of competing perspectives and bring 'some degree of comfort and certainty to us and our students' (Poole, 2009, 51). Without this comfort and certainty, these academically successful students developed techniques for gaining clarity on assessment, most of which involved dialogue with both tutors and fellow students about their work prior to submission. Students were prepared to use any effective tactic to tease out the diverse assessment expectations of their multidisciplinary degree, and indeed any inconsistencies between individual assessors. Once assessment expectations had been understood, or at least were perceived to be understood, then students faced the challenge of meeting them. As one student suggested 
switching gears from writing a Foucauldian essay in one field of study to a business report in another requires an agility that is challenging to enact.

Faced with accounts of such a fragmented experience it could be suggested that practitioners should aim to enhance coherence across a multidisciplinary course, however, this is challenging in practice. Moore reflects that this requires a ' $h i g h$ level of ideological consensus among the staff' (Moore 2000,187) involving academics affiliated to a course to agree on what counts as valid knowledge, and why and how it is to be recognised. Moore $(2000,188)$ describes this as requiring a shared 'social epistemology of the curriculum'. Popkewitz \& Brennan $(1998,9)$ define social epistemology as moving beyond epistemological essentialism to 'embody historically constructed values, priorities and dispositions toward how one should see and act towards the world'. However, it does seem unlikely that academic staff can achieve this across a multidisciplinary curriculum. Within a world context in which the value of cross-disciplinary working is increasingly recognised ironing out discrepancies is perhaps neither realistic nor helpful. Divergent views enrich our understanding of the world and arguably should be viewed as an opportunity not a problem even within a single, coherent discipline. It perhaps behoves us as academics to recognise our own disciplinary beliefs and the ways these are embedded in our assessment practices and judgements and appreciate the different ways of knowing and practicing of others at least within the fields of study we teach.

Although it was not an explicit objective of this study it is perhaps worthy of mention that all four student research assistants graduated with first class honours (only one had been predicted to do so prior to the research). Informally, the SRAs discussed the benefits of participation in the 
research project particularly in terms of understanding the assessment challenges inherent within a multidisciplinary context and intentionally determining some ways of addressing these. Seale (2010) suggests there are other benefits to be drawn from participatory research with students. Participants are encouraged to own the outcome of research by setting the goals and sharing in decisions about processes within less hierarchical relationships in which researcher and participant have more equal status and power. Indeed, the SRAs felt empowered to drop into the offices of the research academics to discuss not only the research project but also the challenges and successes of their own dynamic learning experience. But whilst in this project efforts have been made to democratise the research process, we must be cautious in any claim for true student empowerment. As Fielding $(2004,309)$ comments 'there are no spaces, physical or metaphorical, where students and staff meet one another as equals, as genuine partners in shared undertaking of making meaning of their work together'. Findings presented here reflect an attempt to co-create meanings with students, but we make no claims for true emancipatory research collaboration. Due to writing and publication timelines, all the SRAs had graduated well before this paper was written or published and felt unable to engage in the co-creation of what was viewed as 'another assignment' from a former context.

\section{Conclusion}

The rhetoric is that the world needs more people capable of working in multidisciplinary contexts (Feng 2012). Multidisciplinary degrees have the potential to provide a learning environment within which the ability to recognise and work within different ways of knowing is intentionally developed. However, this research highlights the intensity of the learning and assessment challenges that students experience studying across departments and disciplines. 
Assessment understood as situated and interpreted is embedded in the epistemic assumptions and educational values of learning communities (Shay 2008). Students experience divergent approaches to assessment both within and across disciplines, which problematizes the development of their assessment literacy. It is unlikely that course teams will achieve the ideological consensus required to gain consistent viewpoints on valid ways of knowing including assessment literacy across a programme. Accordingly it perhaps behoves teaching academics, not only to explicitly recognise and acknowledge the varied epistemic assumptions in play in our programmes, but also to intentionally develop and share strategies to communicate this variation to students, not with a view to achieve epistemological and ideological consistency but for students to better understand, appreciate and cope with variation. 


\section{References}

Becher, T. and P. Trowler. 2001. Academic tribes and territories: intellectual enquiry and the culture of disciplines. $2^{\text {nd }}$ ed. Buckingham: The Society for Research into Higher Education.

Bergold. J. and S. Thomas. 2012. "Participatory Research methods: A methodological approach in motion. Forum: Qualitative Social Research."13(1): doi:10.17169/fqs-13.1.1801

Bernstein, B. 1996. Pedagogy, symbolic control and identity: theory, research, critique. London: Taylor and Francis.

Biglan, A. 1973a. "The characteristics of subject matter in different scientific areas." Journal of Applied Psychology 57(3): 195-203. doi: 10.1037/h0034701.

Biglan, A. 1973b. "Relationships between subject matter characteristics and the structure and output of university departments." Journal of Applied Psychology 57(3): 204-213. doi: $10.1037 /$ h0034699.

Boyd, B. and C. Smith. 2016. "The contemporary academic: orientation towards research work and researcher identity of higher education lecturers in the health professions." Studies in Higher Education (41)4: 678-695. doi.org/10.1080/03075079.2014.943657

Braxton, J.M. \& Hargens, L.L. 1996. "Variation among academic disciplines: analytical frameworks and research." In Higher Education: handbook of theory and research, edited by J.C. Smart. 1-46. New York: Agathon Press

Canning, J. 2005. "Disciplinarity: a barrier to quality assurance? The UK experience of area studies." Quality in Higher Education 11(1): 37-46. doi:10.1080/13538320500074931

Dean, A. and P. Gibbs. 2015. "Student satisfaction or happiness? A preliminary rethink of what is important in the student experience." Quality Assurance in Education 23(1): 5-19. doi:10.1108/QAE-10-2013-0044.

Feng, L. 2012. "Teacher and student responses to interdisciplinary aspects of sustainability education; what do we really know?" Environmental Education Research 18(1): 31-43. doi: 10.1080/13504622.2011.574209

Fielding, M. 2004. "Transformative Approaches to Student Voice: Theoretical Underpinnings, Recalcitrant Realities.” British Educational Research Journal 30(2): 295 -311. doi: 10.1080/0141192042000195236.

Gibbs, G. and Simpson, C. 2005. "Conditions under which assessment supports student learning." Learning and Teaching in Higher Education 1:3-31.

Hughes, G., H. Smith, and B.Creese. 2015. "Not Seeing the Wood for the Trees: Developing a Feedback Analysis Tool to Explore Feed Forward in Modularised Programmes." Assessment and Evaluation in Higher Education 40 (8): 1079-94. doi 10.1080/02602938.2014.969193 
Goodyear, P. and R. Ellis. 2007. "The development of epistemic fluency: Learning to think for a living." In Transforming a university: the scholarship of teaching and learning in practice, edited by A. Brew and J. Sachs, 57-68. Sydney: Sydney University Press.

Jones, A. 2007. "Multiplicities of manna from heaven? Critical thinking and the disciplinary context." Australian Journal of Education 51(1): 84-103. doi: 10.1177/000494410705100107

Klein, J.T. 1990. Interdisciplinarity: History, Theory, and Practice. Detroit: Wayne State University Press.

Kvale, S. 1996. An introduction to qualitative research interviewing. Thousand Oaks: Sage.

Lattuca, L. 2001. Creating Interdisciplinarity. Nashville: Vanderbilt University Press.

Lea, M. and B. Street. 2006. "The Academic Literacies Model: Theories and Applications", Theory into Practice 45(4): 368-377.

Macfarlane, B. 2011. "The Morphing of Academic Practice: Unbundling and the Rise of the Para-academic." Higher Education Quarterly 65(1): 59-73. doi: 10.1111/j.14682273.2010.00467.x

Mathieson, S. 2012. "Disciplinary cultures of teaching and learning as socially situated practice: Rethinking the space between social constructivism and epistemological essentialism from the South African experience." Higher Education 63(5): 549-64. doi: 10.1007/s10734-011-94583

Moore, R. 2000. "The (Re) Organisation of Knowledge and Assessment for a Learning Society: the constraints on interdisciplinarity." Studies in Continuing Education 22(2):183-199. doi: $10.1080 / 713695730$

Morrison, D., and A. Collins. 1996. "Epistemic fluency and constructivist learning environments." In Constructivist learning environments, edited by B. Wilson, 107-119. Englewood Cliffs, NJ: Educational Technology Publications.

Neumann, R., S. Parry, S., T. Becher. 2002. Teaching and Learning in their Disciplinary Contexts: A conceptual analysis. Studies in Higher Education 27(4): 405-417. doi: $10.1080 / 0307507022000011525$

O’Donovan, B. 2017. "How students beliefs about knowledge and knowing influence their satisfaction with assessment and feedback." Higher Education, 74(4): 617633. doi:10.1007/s10734-016-0068-y

O'Donovan, B., M. Price, M., C. Rust. 2004. "Know what I mean? Enhancing student understanding of assessment standards and criteria." Teaching in Higher Education 9(3): 325-336, doi:10.1080/1356251042000216642

Paulsen, M. B., and C.T. Wells. 1998. "Domain differences in the epistemological beliefs of college students." Research in Higher Education 39(4): 365-384. doi: 10.1023/A:1018785219220

Poole, G. 2009. “Academic Disciplines: Homes or Barricades?” In The University and its Disciplines, edited by C. Kreber. 50-58. New York: Routledge.

Popkewitz, T. and M. Brennan, eds. 1998. Foucault's challenge: Discourse, Knowledge and Power in Education. New York: Teachers College Press. 
Price, M., C. Rust, B. O’Donovan, K. Handley, with R. Bryant. 2012. Assessment Literacy: The Foundation for Improving Student Learning. Oxford: OCSLD

Ramsden, P. 1992. Learning to Teach in Higher Education. London: Routledge.

Sadler, D. R. 1989. "Formative assessment and the design of instructional systems." Instructional Science 18(2): 119-144. doi: 10.1007/BF00117714.

Sadler, D.R. 2009. "Indeterminacy in the use of preset criteria for assessment and grading in higher education." Assessment and Evaluation in Higher Education, 34(2): 159-179. doi:10.1080/02602930801956059

SCUP Academy Council (2014) Report on Trends in Higher Education Planning 2014. Ann Arbor: Society for College and University Planning (SCUP).

Seale, J. 2010. "Doing Student Voice Work in Higher Education: An Exploration of the Value of Participatory Methods. ” British Educational Research Journal 36(6): 995 - 1015. doi: $10.1080 / 01411920903342038$

Shay. S. 2008. "Assessment at the boundaries: Service learning as case study." British Educational Research Journal 34(4): 525-540. doi: 10.1080/01411920701609406

Timmis S., and J. Williams. 2017. "Playing the interdisciplinary game across education-medical education boundaries: sites of knowledge, collaborative identities and methodological innovations." International Journal of Research \& Method in Education 40(3): 257-269, doi: 10.1080/1743727X.2017.1299125

Trowler, P. 2014. "Depicting and researching disciplines: strong and moderate essentialist approaches." Studies in Higher Education 39(10): 1720-1731. doi: 10.1080/03075079.2013.801431

Vaughan, K. 2015 "Pieced together: Collage as an artist's method for interdisciplinary research." International Journal of Qualitative Methods (4)1: 27-52. doi:10.1177/160940690500400103

Weissmann, E. 2013. "Excluded from the institutional habitus: the joint student experience." Journal of Further and Higher Education 37(2): 261-279. doi: 10.1080/0309877X.2011.645451

Weller, S., G.K. Domarkaite, C. Lam, L. Metta. 2013. "Student-faculty co-inquiry into student reading: Recognising SoTL as pedagogic practice." International Journal for the Scholarship of Teaching and Learning 7(2): 1-16. doi: 10.20429/ijsotl.2013.070209

Welikala, T and C. Atkin. 2014. "Student co-inquirers: the challenges and benefits of inclusive research". Journal of Further and Higher Education 37(2): 390-406. doi: 10.1080/1743727X.2014.909402

Wittgenstein, L. 1953. Philosophical investigations. London: Blackwell Publishing. 